Página inicial: 571 - Página final: 590

Tipo de artículo: Reflexión derivada de investigación

\title{
Consideraciones para la construcción crítica del currículum y la didáctica en los humanismos. ${ }^{i}$
}

Considerations for the critical construction of the curriculum and didactics in Humanism.

Recibido: noviembre 2015 Revisado: abril 2016 Aceptado: mayo 2016

Por: Fabián Alonso Pérez Ramirez ${ }^{1}$, Lizeth Palacio Rios ${ }^{2}$, German Dario Hernández Zuluaga ${ }^{3}$

\section{Resumen.}

Se plantean los componentes de un currículo crítico en filosofia, ética y religión, partiendo de una aproximación reflexiva a la didáctica y el currículum. Los resultados permitieron develar tres tendencias en los diseños curriculares: historicista, estandarizado y problematizador, que orientan las prácticas formativas en los humanismos, así mismo, se evidencian las experiencias pedagógicas significativas tales como: la disertación filosófica, los encuentros de oratoria, el foro intercolegial de filosofia, el uso de las tecnologías de la información y la comunicación aplicadas a la formación ética y religiosa, todas, asociadas a diversos enfoques didácticos. Finalmente, se presenta la construcción de un currículo crítico octagonal y la posibilidad abierta de plantear diversas cartografias curriculares, según cada contexto institucional, como propuesta de innovación educativa y consolidación creativa del colectivo docente de la Mesa de Humanismos.

\section{Palabras claves.}

Didáctica crítica, currículum crítico octagonal, Mesa de Humanismos.

\begin{abstract}
.
The components of a critical curriculum in philosophy, ethics, and religion, are considered, on the basis of a reflective approach to didactics and the curriculum. The results allowed to unveil three trends in curriculum designs: historicist, standardized and problematic, which guide the educational practices in Humanisms. Likewise, significant pedagogical experiences such as philosophical dissertation, public speaking meetings, the philosophy intercollegiate forum, the use of information and communication technologies applied to the ethical and religious education, are highlighted, all of which are associated with various didactic approaches.
\end{abstract}

\section{Key words.}

Critical Didactics, Critical Curriculum Octagonal, Humanism Committee.

1 Estudiante del Doctorado en Ciencias de la Educación de la Universidad San Buenaventura (USB) Medellín. Magister en Educación, Especialista en Pedagogía y Didáctica, Licenciado en Filosofia y Ciencias Religiosas de la Universidad Católica de Oriente (UCO). Docente-investigador del Grupo Servicio Educativo Rural (SER) de la Universidad Católica de Oriente (UCO), Facultad de Ciencias de la Educación. Rionegro, Antioquia, Colombia. fperez@uco.edu.co

2 Licenciada en Filosofia y Educación Religiosa. Auxiliar de investigación en el proyecto "Construcción de un currículo critico en filosofia, Educación Religiosa y Ética - Municipio de Rionegro Antioquia”. Universidad Católica de Oriente (UCO). Rionegro, Antioquia, Colombia. Correo electrónico: lizparios@gmail.com

3 Licenciado en Filosofia y Educación Religiosa. Auxiliar de investigación en el proyecto "Construcción de un currículo critico en filosofia, Educación Religiosa y Ética - Municipio de Rionegro Antioquia”. Universidad Católica de Oriente (UCO). Rionegro, Antioquia, Colombia. Correo electrónico: timotheosevangelos@gmail.com 


\section{Introducción.}

Históricamente, la didáctica ha devenido de ser un género literario en la época clásica (siglo IV a.C.), a configurarse como una disciplina general de la enseñanza en el siglo XVII y las discusiones que la acompañan del siglo XX a la fecha, con pretensiones de una disciplina autónoma. Actualmente, se puede plantear una fuga didáctica que va de teorías diafragmáticas (transposición didáctica y asociaciones con las epistemologias de las ciencias), hasta las meta-teorías (mega-relatos de las disciplinas).

Se identifican así, diversos enfoques didácticos, a saber: una didáctica general, heredera de las ciencias de la educación de la tradición francófona; una didáctica diferencial que congrega visiones psicologistas y neurológicas; unas didácticas de las ciencias, que aglomeran las epistemologias, lógicas y métodos de dichas ciencias; y finalmente, una didáctica crítica reconstructiva, heredera de la tradición alemana cuya intencionalidad es develar las ideologías y formas de reproducción de proceso formativo. (Camilloni, Divini, Edelstein, Litwin, Souto \& Barco, 1997)

Desde los planteamientos anteriores, la didáctica como disciplina, se ha asumido desde visiones de tipo instrumentalista, con una fundamentación epistemológica de las ciencias y la psicología que la reducen al positivismo y funcionalismo. Es así como desde “(...) campos específicos de las respectivas ciencias, sin relación con un marco de didáctica general cuya propia existencia se cuestiona, desde la óptica de que la enseñanza siempre opera sobre contenidos de instrucción especializados (Camiloni, et al, 1997, p. 42-43)

Esta concepción del ejercicio didáctico en la enseñanza, ha centrado su interés en los mecanismos de producción y la cuantificación del saber, relegando la imperante necesidad de preguntarse por los valores, por el contexto y por la cualificación de la formación humana. Sin embargo, hoy se puede considerar la didáctica no sólo desde este enfoque tecnologizante, sino como una disciplina teórico - crítica, que trasciende su instrumentalidad. En cuanto teoría critica, es importante precisar, que ésta se refiere a una teoría social filosófica que nace en los años veinte en Alemania. Algunos teóricos como Theodor W. Adorno, Max Horkheimer, Herbert Marcuse y Jürgen Habermas, colaboraron en el marco de la conocida Escuela de Frankfurt y elaboraron teorias que influyeron decisivamente en la configuración de las ciencias sociales a partir de los años setenta.

La ciencia de la educación -nótese la singularidad y no la pluralidad del término-, incorpora también estas tesis de la teoría critica en su reflexión pedagógica, y en lo que concierne a la didáctica, es Wolfgang Klafky, principalmente, quien relaciona estos postulados para una concepción trascendental de la ciencia de la educación -o pedagogía- como teoría critico - constructiva, donde la actividad mental propia de los sujetos y por ende el aprendizaje, responde a contextos biológicos y sociales.

Sin la actividad mental propia de cada sujeto que obedece a las necesidades vinculadas al desarrollo evolutivo y socio-histórico, es imposible que el participante de un proceso educativo se apropie de los conocimientos suficientes para la comprensión y transformación de la realidad natural y socio-histórica y, por tanto, no solo se limita el acceso al bienestar y calidad de vida, sino a su realización como ser humano (Arias, Gómez, \& Osorio, 2008, p. 35)

Esta nueva concepción sobre la enseñanza y el aprendizaje supone, que la didáctica no se reduce meramente a cuestiones instrumentales, ni a la pregunta por el cómo, pues también 
debe tener presente la reflexión que aborda otros interrogantes, la acción que reconstruye el conocimiento, que articula la lectura del contexto y que se encuentra en el horizonte de una racionalidad emancipadora individual y social.

Entiendo la ciencia de la educación en general y la didáctica en especial, como una teoría crítico-constructiva. Dicho brevemente, se trata de una teoria relacionada con la praxis, que se sirve de planteamientos y métodos hermenéuticos, empíricos y critico-ideológicos y por otra parte de una teoría que no permanece en la simple descripción e investigación de regularidades o causalidades, ni tampoco en la indicación hermenéutica ni el análisis crítico de las condiciones o presuposiciones, sino que avanza hacia propuestas constructivas a partir de la investigación de la praxis didáctica presente y de las propuestas teóricas existentes ( Klafki, 1991, p.1)

Es por esto que las consideraciones de una didáctica crítica en los humanismos, implican un cambio de paradigma en relación con la praxis y las teorias que intentan definir desde la descripción la comprensión de los procesos de la enseñanza y el aprendizaje, para asumir el reto en la comprensión de las prácticas formativas desde propuestas constructivistas, planteadas por los docentes, para "contemplar a los profesores como profesionales reflexivos de la enseñanza" (Giroux, 1997, p.5) y reconstruirlas con los estudiantes y las comunidades educativas en cada caso.

Estas consideraciones para la construcción crítica del currículum y la didáctica en los humanismos, implica unas aclaraciones mínimas sobre los limites, articulaciones y tensiones existentes entre la didáctica y el curriculum. Lo primero que hay que decir, es que en su origen ambas responden a tradiciones pedagógicas diferenciadas. Mientras la didáctica como disciplina, emerge en la tradición francófona - Mialaret - (de las ciencias de la educación), el currículum nace en la tradición anglosajona.

La tradición anglosajona de los Curriculum Studies es la más reciente de las tres tradiciones y, sin embargo, la más influyente y extendida. Sus inicios remontan a mediados del siglo xix con las elaboraciones de ingleses como Herbert Spencer, pero su desarrollo se dio a partir de las primeras décadas del siglo xx, particularmente en los Estados Unidos de América, y su propagación más allá de los límites norteamericanos es producto, fundamentalmente, del movimiento de mundialización de la educación iniciado después de la segunda guerra mundial (Martínez; Castro; Noguera, 2003, p. 48).

Los hilos de la historia han tejido tensiones entre el currículum y la didáctica, reconociendo en ambos diversos enfoques y, cómo cada uno de ellos implica un tipo de relacionamiento. Por ejemplo, un currículum de enfoque técnico presenta un vínculo directo con una didáctica positiva instrumental. Mientras el primero piensa contenidos, temas y áreas del conocimiento, el segundo acoge la enseñanza desde lógicas, métodos, visiones psicológicas y epistemológicas de los campos del conocimiento en cuestión.

Por su parte, las tensiones dadas entre un currículum y una didáctica de enfoques críticos se da en términos de: primero, el reconocimiento en perspectiva histórica del desarrollo de estos dos campos de reflexión pedagógica, que problematicen sus orígenes, alcances, desarrollos y tensiones en la construcción de sentidos e intencionalidades formativas. Segundo, la visibilización de las luchas políticas implícitas y explícitas que se dan en esos diferentes enfoques curriculares y didácticos, en la triada: prácticas didácticas y curriculares, sus teorías y la normatividad que intenta regularlas. Y tercero, las relaciones 
entre los sujetos, los contextos, los saberes y los poderes en clave didáctico - curricular, que amplían no sólo su campo teórico y reflexivo, sino práctico y transformativo.

En este sentido, el currículo que orienta los humanismos no debe ignorar el contexto y exige una formación que suscite niveles de análisis, crítica y alternativas de comprensión para la transformación. Este tipo de formación promueve la emancipación desde la identificación de las problemáticas propias del contexto. Esto implica que el maestro de humanismos asuma su práctica a partir de una caracterización y concienciación de las necesidades de sus estudiantes, a través de preguntas problematizadoras, técnicas interactivas y diagnósticos participativos, que fomenten el pensamiento crítico - emancipador.

Es así como la indagación por el diseño curricular que orienta las prácticas pedagógicas en los campos de formación del humanismo (Filosofia, Educación Religiosa y Ética) tanto en la básica como en la media en el municipio de Rionegro-Antioquia-Colombia, dan cuenta de unos contextos, retos, oportunidades y necesidades que se manifiestan en las realidades de las Instituciones Educativas, desde los estudiantes y los maestros que asumen la formación humana en estos campos disciplinares, en todos los casos con fortalezas y dificultades de diversa índole; lo que da cuenta del acto pedagógico como un escenario de luchas, confrontaciones, intereses, negociaciones culturales y formabilidad humana.

\section{Método.}

El enfoque de la investigación fue cualitativo, entendida como el "esfuerzo por comprender la realidad social como fruto de un proceso histórico en construcción visto a partir de la lógica y el sentir de sus protagonistas" (Sandoval Casilima, 2002, p. 11) cuya modalidad fue investigación-acción, la cual se remonta a los planteamientos de Kurt Lewin. "Este autor fue el primero en utilizar la expresión "investigación-acción" (Hurtado, 2010, p. 603). La investigación acción devela las relaciones que se establecen entre lo social y la formación de los sujetos, las formas de reproducción cultural o de transformación y creación de las relaciones sociales, las manifestaciones del poder, la educación y la construcción crítica de la conciencia para la emancipación.

\section{Participantes, estrategia y algunas aclaraciones.}

En relación con el tipo de muestra, esta es significativa, no probabilística, permitiendo identificar desde la diversidad de la población, su heterogeneidad, (más allá del porcentaje de participación, en relación con la población total), dado el enfoque cualitativo, su paradigma crítico-social y la modalidad de investigación-acción, se conforma y consolida con 15 maestros del municipio de Rionegro-Antioquia, que voluntariamente se vincularon al proyecto, al servir las áreas en mención y participar activamente de los encuentros en la Mesa de Humanismos (estrategia), dinamizando la construcción colectiva del currículo crítico octagonal.

Una mesa de trabajo de maestros es un conversatorio permanente de reflexión, construcción colectiva y socialización de teorías y prácticas educativas, según las disciplinas de formación en cuestión, para la consolidación de redes académicas que articulen elementos pedagógicos, didácticos, curriculares, disciplinarios, investigativos y evaluativos (Pérez, 2014, p.4). 
Siendo la mesa la estrategia, se hace fundamental aclarar la referencia a los humanismos con la intención de dar luces en su comprensión integrativa, desde la cual se identifican los campos de filosofia, ética y educación religiosa, vinculadas a una tradición que cultiva la configuración de lo humano. El término humanismo se devela en los estudios clásicos, como se ve a continuación

Al parecer, fue F.I. Niethammer quien lo utilizó por vez primera para indicar el área cultural a la que se dedican los estudios clásicos y el espíritu que les es propio, en contraposición con el área cultural que cubren las disciplinas científicas. No obstante, el término "humanista" (y sus derivados en las diversas lenguas) nació hacia mediados del siglo xv, inspirado en los términos "legista", "jurista", "canonista", o "artista", para indicar a quienes enseñaban y cultivaban la gramática, la retórica, la poesía, la historia y la filosofia moral. Además, en el siglo xiv ya se había hablado de Studia humanitatis y de studia humaniora, citando afirmaciones famosas de Ciceron y de Gelio, para señalar tales disciplinas (Reale y Antiseri, Tomo II, 1998, p. 26).

Se vislumbra que las disciplinas científicas en su intento por comprender lo humano, dado su pragmatismo, lenguaje técnico y métodos, se quedan cortas a la hora de abordar tal complejidad. De ahí la necesidad de asumir que, el humanismo como campo interdisciplinar, sea uno de los caminos propicios para la aproximación y configuración de aquello que se denomina humano.

En este sentido, tales aproximaciones y configuraciones de lo humano exigen una mirada y unos lenguajes críticos. Esto implica un lugar desde el cual se deconstruye y construye el pensamiento mismo, sus alcances e intencionalidades emancipatorias, donde ser, pensar y poder constituyen la triada que abre el horizonte del entendimiento para la transformación. Estas perspectivas críticas "nos enseñan a sentir y a pensar en y con el otro, mueven y a la vez nos colocan ante las realidades que nos constituyen" (Zemelman, 2012, p. 9).

Somos a través de la acción comunicativa y ella nos determina, junto a los contextos, los tipos de relaciones entre los sujetos y el mundo, ya sean estas emancipatorias o de opresión. Se trata entonces del desocultamiento de las formas de poder que existen en el conocimiento y por ende en los sujetos y sus relaciones. De ahí que "un desafio que pone en cuestión la estructura simbólica del mundo de la vida en su totalidad, explique por qué éstas nos han vuelto accesibles precisamente a nosotros" (Habermas, 2008, p. 572).

Finalmente, la coherencia que ha de existir entre los participantes (maestros coinvestigadores), la estrategia (Mesa de Humanismos) y la perspectiva critica (teórica-metodológica), consolidan las bases sobre las cuales se orientó la investigación, poniendo en tensión los sujetos-contextos-saberes-poderes con la posibilidad de una praxis inquietante, donde la educación es uno de los escenarios por excelencia, no sólo de conservación de la cultura, sino y por ende de su transformación.

\section{Técnicas.}

La investigación asumió la observación participante y no participante (durante todo el proceso). El análisis documental se realizó por medio de matrices, las cuales permitieron visualizar los diseños curriculares que orientan a los docentes en sus procesos de formación y develar los enfoques y tendencias curriculares que los configura. Tres grupos focales -uno por cada campo en cuestión- donde se recuperaron experiencias pedagógicas significativas desde elementos contextuales, pedagógicos, didácticos, curriculares y evaluativos, con el fin 
de ser documentadas y valoradas como saber pedagógico construido por los docentes, que develan enfoques didácticos. "Los grupos focales son una técnica de recolección de datos mediante una entrevista grupal semiestructurada, la cual gira alrededor de una temática propuesta por el investigador" (Escobar \& Bonilla, 2011, p. 2).

Una vez realizados los grupos focales se procede a la sistematización de los datos obtenidos y como siempre, su sintesis fue sometida a la validación, siendo esta un ejercicio de legitimación de las descripciones y análisis que se logran por medio del debate, el contraargumento y la valoración que los co-investigadores (los 15 maestros que participaron de la mesa) hacen de los constructos individuales y colectivos, con el propósito de llegar a acuerdos intersubjetivos y consensos que acojan todas las voces, donde no siempre existen sintesis que abrazan fraternalmente las tesis y antitesis, sino que también hay espacio para que las tesis y antitesis queden abiertas a nuevas discusiones y validaciones.

Finalmente se realizaron tres talleres denominados: Construcción colectiva de un currículo crítico en filosofía, ética y religión, por campos específicas de formación en los humanismos, dando forma a la propuesta del currículo octagonal, que se va consolidando colectivamente con la comunidad académica pensante de maestros, tomando como referentes sus vivencias, el contexto, las preguntas y el sentido formativo visto desde las experiencias con sus grupos; se indaga además por la relación de estos elementos del contexto con los componentes disciplinares de cada área, que en el apartado de los hallazgos trataremos más ampliamente. Esta es una de las técnicas interactivas que responde a las tradiciones de la investigación social, cualitativa.; el taller "es un espacio de construcción colectiva que combina teoria y práctica alrededor de un tema, aprovechando la experiencia de los participantes y sus necesidades de capacitación” (Candelo, Ortiz \& Bárbara 2003, p. 33).

Figura 1. Que relaciona 5 componentes de reflexión crítica en el campo pedagógico

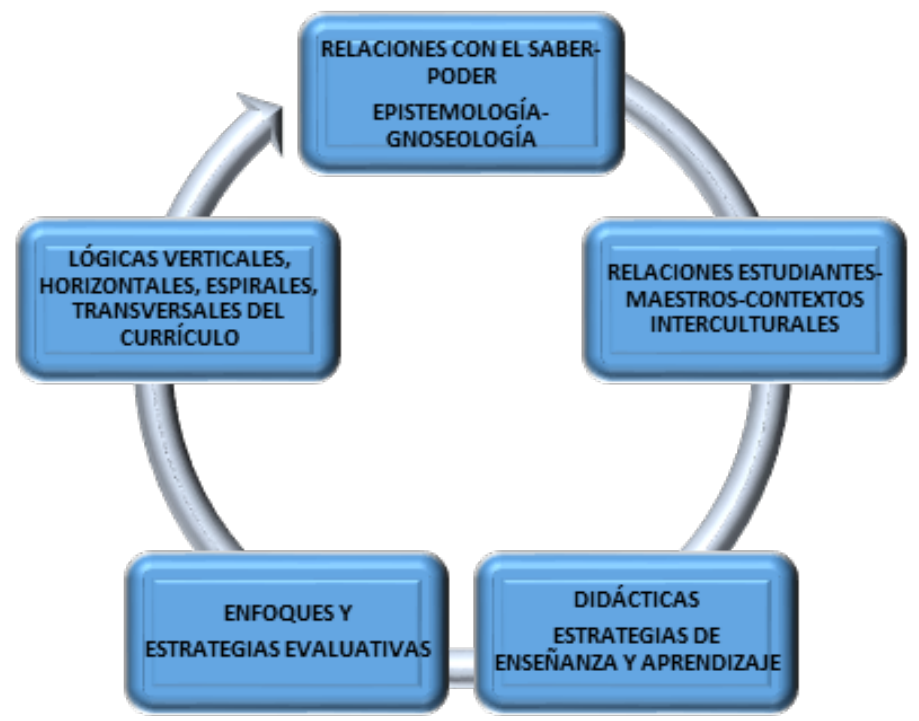

Fuente: Elaboración propia con base en el análisis crítico del campo pedagógico con los maestros 
Figura 2. Currículo octagonal, que relaciona los campos problematizadores de un currículum crítico.

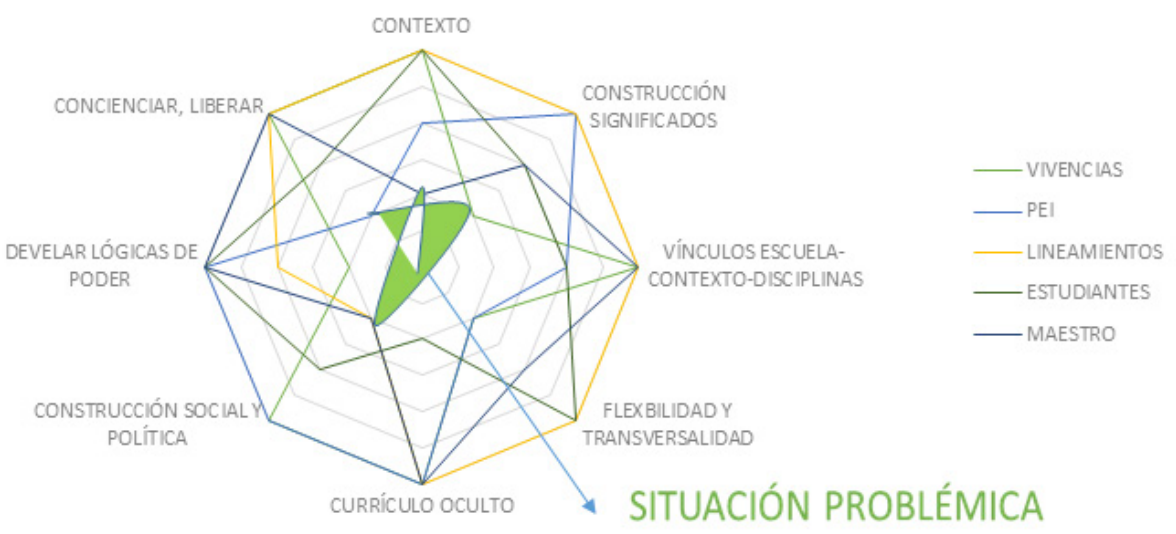

Fuente: Elaboración propia con base en el análisis de los campos de problematización curricular.

\section{Resultados.}

\section{Hallazgos a partir del Análisis de las Mallas Curriculares.}

En los diseños de las mallas en Filosofia, Ética y Educación Religiosa en la básica secundaria y la educación media, es notorio un ejercicio de homogenización en perspectiva de lógica vertical (donde se asumen los componentes generales y estructurantes de las mallas, presentando diversidad en las nominaciones, que ya de por sí, indican enfoques curriculares, pero recurrentes en lo que señalan), y una lógica horizontal (donde relacionan la dimensión temporal en bloques, según los periodos académicos en cada Institución estipulados), que presentan las matrices donde se diseñan los micro-currículos en cada área y de cada institución Educativa, con algunos rasgos diferenciadores en tanto los componentes que se identifican. Es así como se aprecian tres grandes tendencias en los enfoques curriculares, hasta ahora analizados desde la Mesa de Humanismos.

En primer lugar, se puede hablar de la visión de un currículo de corte historicista. E1 desarrollo de este currículo se aborda por periodos académicos en donde prima la información histórica sobre el área. En el campo de la filosofia, cada periodo está programado para abordar una época diferente de su historia; en el campo de la educación religiosa se aborda la historia desde el componente bíblico-eclesial y/o la historia de las religiones desde enfoques culturalistas, mientras en ética, se identificaron temáticas en relación con los proyectos de vida, entendidos como una genealogia, una historia de vida personal por descubrir.

Este currículo con tendencia histórico-hermenéutica permite identificar en sus diseños elementos tales como -núcleos conceptuales, logros, indicadores de logro, estrategias de enseñanza, estrategias de evaluación- señala en sus descriptores una fuerte tendencia a incorporar elementos de las disciplinas en cuestión (filosofia, ética y educación religiosa) desde una mirada metafisica, de abstracciones y generalizaciones frente a la forma de abordar los diseños curriculares y sus acciones formativas. Por un lado abunda el discurso del maestro, 
los meta-relatos, las frases célebres; por el otro, y desde las estrategias de enseñanza y de evaluación, se plantean discusiones en equipos, trabajos de reflexión individual, y lecturas de textos especializados, favorables a la construcción de ensayos e informes de lectura, con la intención formativa de generar comprensiones e interrelaciones entre campos disciplinares, con los otros, entendidos como otras interpretaciones, en ejercicios de construcciones de sentido frente a un núcleo conceptual que busca resignificaciones y ampliaciones del campo semántico, un currículo educativo como práctica interpretativa.

En segundo lugar se encontró la visión de un currículo estandarizado; este tipo de currículo se desarrolla por núcleos establecidos, que por lo general no dan pie a la tematización de los campos de formación en cuestión. Se evidencia de manera clara, la aplicación de un currículo netamente instrumental y unidireccional.

Un currículo de tendencia técnica, en el que según los diseños se plantean: ámbitos temáticos, contenidos, estándares, competencias, objetivos, actividades, técnicas, instrumentos, recursos, fechas que dan cuenta en sus descriptores de intencionalidades predeterminadas, que guian la experiencia de aprendizaje de arriba hacia abajo (estandarización de la enseñanza), donde no se identifican problemáticas propiamente dichas, sino temas y contenidos, que responden y como lo indica Shirley Grundy (1991) al control y manipulación del ambiente, no sólo en el diseño curricular, sino en la ejecución de los currículos en perspectiva unidireccional, donde la intencionalidad formativa está fuertemente guiada por el desarrollo de unos temas no negociables, la implementación de actividades, técnicas e instrumentos de medición de la información apropiada y la puesta en escena de pruebas estandarizadas para la ejecución y comprobación de dicha apropiación, un currículo educativo como producto.

Y en tercer lugar se encontró la visión de un currículo problematizador con rasgos críticos. Este tipo de currículos están planteados por medio de ejes generadores o preguntas problematizadoras que dejan abierta la posibilidad del diálogo y la construcción de los sujetos en formación, es decir, hay una mejor interacción entre el estudiante, los docentes, el contexto y el conocimiento; este tipo de estrategias van formando y fortaleciendo el pensamiento crítico.

Este enfoque presenta aproximaciones a una tendencia de currículo crítico, en el que se encuentran ejes generadores, preguntas problematizadoras, estrategias pedagógicas y didácticas, indicadores de desarrollo, estrategias de evaluación y criterios de evaluación; desde los descriptores se identifican elementos de construcción colectiva en los diseños y desarrollos del currículo (maestros y estudiantes), se promueve la interacción dinámica de la reflexión y la acción, el acto de construir y reconstruir reflexivamente, las formas de comprender, la visibilización de las posibilidades de transformación y las transformaciones propiamente dadas desde las actitudes y los actos, que potencia la autonomía y la coresponsabilidad en la intencionalidad formativa. Desde este enfoque se identifican experiencias de aprendizajes como: las disertaciones, los foros, los debates, las gimnasias y los laboratorios filosóficos, la utilización de redes sociales y las TIC, la lúdica reflexiva, todo esto con el propósito de generar aprendizajes; que identifican un currículo educativo como praxis y dan la posibilidad de perfilar fenomenologías pedagógicas, más allá del enfoque metafisico, de meta-relatos, heredados de una edad media tardía en nuestras tierras. 


\section{Hallazgos desde la Recuperación de Experiencias Significativas.}

La técnica de grupo focal realizada con los maestros de la Mesa de Humanismos, y que tenía como fin la recuperación de sus experiencias significativas, para documentarlas y valorarlas como saber pedagógico, aporta los siguientes hallazgos:

- En cuanto a la lectura de textos, se pone de manifiesto que en algunos casos los docentes se han esforzado por dar trascendencia a dicha lectura, suscitando en los estudiantes una interpretación de textos que no se limita al enfoque hermenéutico, gramatical, sistemático o histórico, sino que trasciende a una lectura crítica, posibilitándole a los estudiantes el poder cuestionar los textos, autores y temáticas, facilitando la potenciación de habilidades para la producción textual filosófica, religiosa y axiológica.

Esta trascendencia en la lectura de los textos se ve claramente evidenciada en experiencias, como la disertación filosófica, donde el estudiante tiene la posibilidad de producir y profundizar un saber a través del modelo de tesis, antítesis y síntesis, actividad que se desarrolla a lo largo del año académico y que finaliza con la socialización al interior de la institución educativa a través de un foro. Frente a esta experiencia dice el maestro participante de la Mesa de Humanismos:

"La enseñanza de la Filosofia en la Educación Media tiene como propósito contribuir a la formación integral del estudiante como persona crítica, creativa y dispuesta al diálogo. Es en aras de satisfacer este objetivo es que se implementa la estrategia didáctica del Foro de Filosofia como un espacio valioso para fomentar el pensamiento crítico, mediante el ejercicio de la disertación filosófica; a través de la cual el estudiante puede exponer una opinión propia y fundada, partiendo de una pregunta problematizadora sobre un tema concreto, para ampliar así el marco de comprensión sobre el mismo" (Carmona, 2012, p. 1).

Esta experiencia está emparentada con una didáctica filosófica, es decir, una didáctica disciplinar especializada (didácticas de las ciencias), erigida desde las lógicas, los métodos y las epistemologías del saber (en este caso el filosófico), cuya pretensión es la enseñanza y aprendizaje de la filosofia a partir de sus propios métodos. A pesar de estas implicaciones didácticas, los efectos curriculares presentan un enfoque crítico, como se lee a continuación.

La lectura de textos también se ha encontrado permeada por un hecho significativo en la formación de los estudiantes, consistente en que los currículos sean ajustados a partir de sus mismas necesidades e intereses. Este hecho implica que el maestro reconozca al estudiante como sujeto generador del currículo, pues al tener en cuenta sus inquietudes e interrogantes, está respondiendo asertivamente a sus necesidades y suscita un mayor interés y receptividad por parte de ellos.

- El foro intercolegial de filosofia es una experiencia que se ha desarrollado durante varios años en el municipio de Rionegro. Es importante resaltar que se ha venido desarrollando de forma articulada con la Mesa de Humanismos, rescatando que las problemáticas a tratar son abordadas desde diferentes paradigmas y enfoques, es decir, religiosos, éticos y filosóficos, posibilitando una articulación de los campos disciplinares y exigiendo la resignificación del saber por medio de la discusión, el análisis documental, la triangulación, el acompañamiento permanente de los maestros, la producción textual y la posibilidad de exponer el pensamiento no sólo a través de la literatura, sino también 
de la música, la poesía, las artes plásticas y el teatro, como otras formas de manifestación del pensamiento. Escribe el maestro:

"Al ser la filosofia cuna de pensamientos insólitos y como se ha dicho siempre una aventura, los jóvenes estudiantes que aceptan el reto para ser ponentes, no solo reciben un tema y se les da un tiempo para realizar un ensayo, sino que, tienen un verdadero acompañamiento en diez encuentros previos en los cuales cuentan con asesores, y con charlas filosóficas qué les sirve en su proceso de formación y escritura" (Ríos, 2014, p. 12)

Se identifica aquí una didáctica de enfoque crítico donde se da un escenario propicio para la construcción y la deconstrucción colectiva de las diferentes formas de pensar, sentir y actuar, complejizando las realidades de los participantes, posibilitando las expresiones de los mismos y abriendo un espacio de reflexión y crítica donde prima la voz de los estudiantes (de los silenciados en las clases), rompiendo con la hegemónica voz del maestro.

- En esta misma línea, pero en la institución Educativa Barro Blanco, quien orienta el área de filosofia, ha realizado durante varios años $(2012,2013,2014)$ una experiencia que se denomina encuentro de oratoria, donde se destaca la participación de aquellos que mayor desempeño tengan en la expresión oral con un tema libre, promoviendo las competencias lingüísticas y el ejercicio de la escucha.

Esta experiencia a primera vista, pareciera responder a un enfoque instrumental de la didáctica, sin embargo, hay que anotar que las intencionalidades formativas de la misma dan cuenta de una didáctica crítica, en tanto que recupera la voz del estudiante, y permite no sólo la expresión oral, sino también la toma de posición ética, política y estética.

- La educación mediada a través de las Tecnologías de la Información y la Comunicación (TIC), es también una experiencia pedagógica que ha tomado valor en la formación humana de los jóvenes. Los maestros se han esforzado por implementar estrategias didácticas que involucran diversas tecnologías de la información y la comunicación, hecho que suscita un mayor interés por parte de los estudiantes, generando nuevas posibilidades de acompañar y aprender de forma cooperativa, las temáticas propuestas en las áreas. En el grupo focal manifestó uno de los maestros que:

"Este proyecto se viene realizando en los grados $8^{\circ}, 9^{\circ}$ y $10^{\circ}$, la idea es trabajar el proyecto de vida articulado a las nuevas tecnologías. Esta propuesta está apenas en su fase inicial, y ya se cuenta con un sitio web desde el cual se pretende la interacción con los alumnos" (Hernández, 2014, p. 3)

Esta experiencia se ha desarrollado principalmente en las áreas de Educación Religiosa y Ética, donde algunos docentes han visto la necesidad de revalorar estas áreas e incentivar a los estudiantes para una participación asertiva y dinámica. Este enfoque didáctico responde a visiones instrumentalistas y funcionalistas, cuyo énfasis sólo logra reflexionar sobre técnicas y estrategias que dinamicen la enseñanza de los saberes.

- En el campo de la educación religiosa, se identificó una experiencia de diseño y práctica curricular; en el texto experiencia de reajustes al plan de área de educación religiosa realizo en la Institución Educativa Gilberto Echeverri Mejía, un maestro manifiesta: 
"El telón de fondo de este plan de área integrado, es propiciar la apertura mental en provecho de una visión cosmopolita en consonancia con las relaciones interculturales de las sociedades contemporáneas que exigen, sin duda alguna, el pluralismo ideológico y religioso. El diseño de este Plan de área propone un desarrollo por etapas semejante al de Piaget, en donde la dificultad temática y la profundidad crítica, incrementa con el proceso educativo año tras año. Alegra, en todo caso que los jóvenes que asisten a las aulas de clase sí aprendan algo" (Ramírez, 2013, p. 1).

En esta experiencia de reajuste curricular, si bien se puede evidenciar una didáctica diferenciada (de enfoque psicologista), el alcance didáctico que implica dicho diseño curricular, se corresponde con una didáctica critica, que permite el diálogo intercultural, visibiliza la diversidad religiosa y las problemáticas de las sociedades contemporáneas en términos de lo sagrado como una construcción de lo humano.

Estas son entre otras, algunas de las experiencias que se identificaron y desde las cuales los docentes vienen dinamizando los procesos formativos, en clave didáctico-curricular, en estos tres campos disciplinares del humanismo.

\section{Hallazgos a partir de los talleres realizados.}

Cada taller tuvo finalidades distintas y permitió que los docentes reunidos por campos específicos, aportaran a la construcción del currículo, desde la observación y la lectura del contexto donde desarrollan su práctica cotidiana. Alrededor de estos ejercicios se manifestó que:

Se han logrado articular las instituciones por medio del trabajo temático Se ha discutido acertadamente problemas del que hacer pedagógico del humanismo. Se ha proyectado el contexto de elaboración de un currículo crítico para la enseñanza de las áreas humanas en Educación Religiosa, Ética y Filosofia (Hernández, 2014, p. 2).

El taller uno pretendió cuestionar los contextos situacionales y cómo esas necesidades o emergencias se ponen en tensión con el campo disciplinar. A partir de su realización se puede afirmar que el diagnóstico situacional de los maestros humanistas se ve permeado por problemáticas externas como las redes de prostitución, la crisis económica, el bullying y ciberbullying, el microtráfico, las familias disfuncionales o no convencionales y una pérdida significativa del interés por los humanismos desde el escenario de las cátedras.

Estas realidades han permitido identificar retos en el campo educativo y social, tales como el desarrollo de un pensamiento crítico y creativo, alternativas de fortalecimiento en la formación, propiciar escenarios alentados por el dinamismo propuesto por los cambios, la sensibilización y promoción de la reflexión.

El taller dos por su parte, tuvo como objetivo que los docentes, a partir de los contextos situacionales y del campo disciplinar, expresaran su concepción con respecto a las estrategias didácticas - metodológicas y de valoración, en correspondencia y pertinencia con los hallazgos del taller uno. "La Mesa de Humanismo ha permitido tener un panorama amplio del ejercicio docente en estas áreas, y conocer metodologías y practicas actuales, que retroalimentan la práctica personal” (Hernández, 2014, p. 2).

Así, los docentes de los humanismos consideran que las estrategias didácticas deben superar métodos tradicionales estandarizados y generar propuestas que sean dinámicas y que 
permitan una problematización por parte de los estudiantes y maestros. Estas estrategias pueden ser debates, videoforos, entrevistas, bitácoras, ludosofias, análisis crítico de textos, proyección social, exposiciones y eventos de integración como el Foro Intercolegial de Filosofia, que permite asumir un enfoque crítico en la didáctica y el currículo.

En el componente valorativo se manifiesta la necesidad de una transformación conceptual y procedimental de la evaluación, superando visiones meramente cuantitativas y trascendiendo a la cualificación y la valoración como proceso. Teniendo en cuenta estos elementos, la valoración se puede desarrollar a partir de la observación de la actitud de los estudiantes, la participación en las actividades, la investigación, las vivencias y la convivencia, las habilidades comunicativas, el análisis contextual y las evidencias de apropiación y consolidación de su pensamiento, en relación con sus afectos y sus actos, al interior del proceso de formación en las áreas.

\section{Ejes a considerar para la construcción del curriculum critico.}

Al momento de construir un currículo critico se hace necesario emanciparse de tradicionales y sesgadas concepciones sobre el currículo, la didáctica y la evaluación, pues el enfoque crítico implica ampliar el espectro y tener en cuenta elementos de orden contextual, que se ven relegados en otras concepciones de didáctica y currículo. En este sentido, la didáctica asumida desde un enfoque crítico trasciende hacia la búsqueda del pensamiento crítico emancipador - transformador.

Estas consideraciones implican una transformación en las reflexiones que se hacen respecto al quehacer educativo, a las relaciones del poder y el saber, el cambio epocal y las emergencias que se convierten en ejes generadores del proceso formativo. Así, la finalidad del proceso de enseñanza - aprendizaje, dado por este enfoque, ya no está orientado a un conocimiento exclusivamente tecnologizante o memorístico, pues éste debe estar dirigido a que el estudiante pueda hacer uso de elementos conceptuales y vivenciales para la proyección social, exigiéndose ser mediador de su propia formación y la de los demás, para menguar y superar las necesidades y problemáticas de su contexto o para potenciar aspectos positivos presentes en el mismo.

El currículo, dentro de este enfoque crítico, exige también una transformación en la concepción conceptual y procedimental, cuestionando las lógicas verticales y horizontales y asumiendo el proceso como espacio de reciprocidad y construcción. Esto supone tener en cuenta elementos que permitan la inclusión de las emergencias del contexto, los deseos y expectativas de los estudiantes y una retroalimentación constante que dé cuenta de la pertinencia del currículo.

El currículo octagonal, paralelamente a como se construye un octágono en la geometría clásica, responde a ocho lados y ocho vértices (los lados corresponden a los componentes del currículo, mientras que los vértices con las tensiones y relaciones que se establecen entre cada componente). Esta figura en el currículo pretende por un lado, decodificar la rigidez en las relaciones fila - columna que se establecen tradicionalmente en los diseños y prácticas curriculares, y por otro lado, configurar una ruta cíclica y en espiral, que, así como responde a la construcción de un octágono en la geometría, se evidencia en los procesos de formabilidad, desarrollo y crecimiento humano, dados por ciclos.

Existe una relación proporcional en la geometría del octágono, en la que la suma de sus ángulos internos (a) es $360^{\circ}$ (una revolución) y la suma de sus ángulos internos (b) es 
$1080^{\circ}$ (tres revoluciones). Esta metáfora en la construcción del octágono implica cuatro revoluciones curriculares: la del saber ser, la del saber, la del saber hacer y la del saber convivir, que se proponen implícitamente en la construcción del currículo octagonal, cuya mediatriz es la concepción y práctica de una pedagogia crítico-social; eje central del octágono, punto equidistante entre los vértices que ponen en tensión los componentes (contextualización, campo disciplinar, mediación, valoración, emergencias, proyección, acción - reflexión y re-contextualización), con el propósito que cada docente en su praxis, en relación con el diseño y realización del currículo octagonal en los contextos educativos, configure según los propios ritmos en el acto formativo (maestros-estudiantes), sus propias cartografias curriculares, sus propias formas de desarrollar los componentes, ponerlos en tensión, reconstruirlos.

Figura 3. Que relaciona los campos contextuales, disciplinares, didácticos y evaluativos

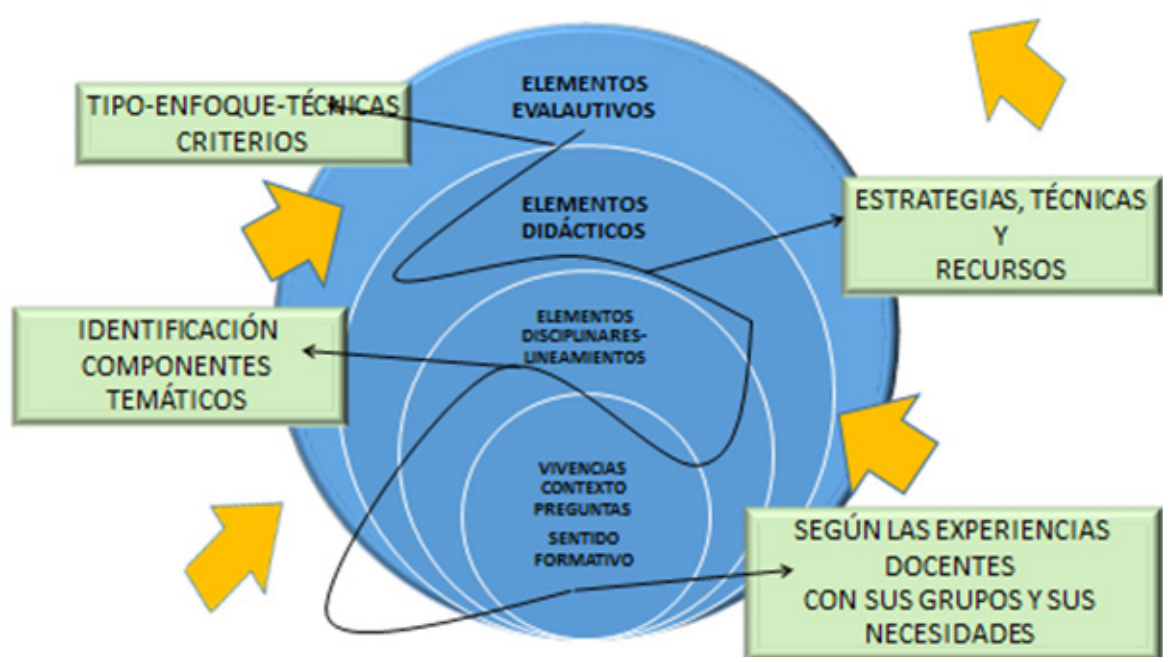

Fuente: Elaboración propia con base en el análisis de los campos de problematización crítica

Figura 4. Propuesta de currículum crítico octagonal

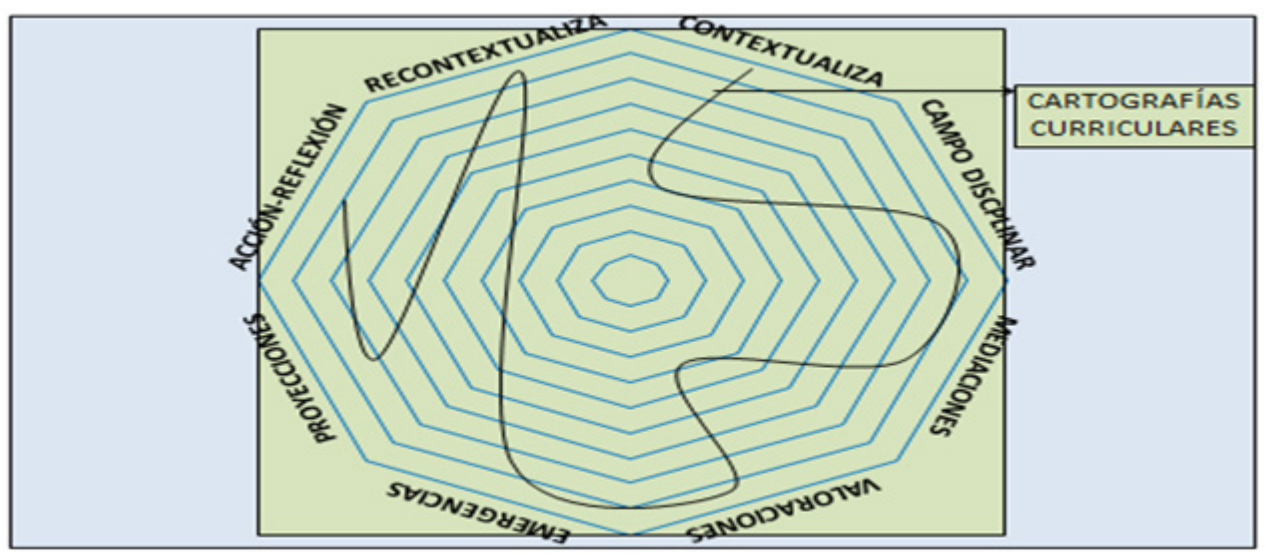

Fuente: Elaboración propia, que emerge de la reflexión crítica y propositiva con los maestros. 


\section{Para la construcción del currículo crítico - octagonal, es necesario considerar:}

- Contextualización: Este componente debe dar cuenta de un análisis diagnósticoparticipativo, que se actualice constantemente y que precise las necesidades formativas y sociales de la población escolar.

- Campo disciplinar: Este componente no puede estar en disociación con el componente de contextualización, pues el currículo exige que las necesidades, problemáticas y potencialidades encontradas en el diagnóstico situacional-participativo, sean puestas en tensión con los campos del conocimiento que se identifiquen y respondan a dichas necesidades-potencialidades.

- Mediaciones: Este componente debe responder al cómo didactizar (y desde qué enfoques) los contextos puestos en tensión con los campos del conocimiento. En este sentido, las estrategias responden al fortalecimiento de las potencialidades y recursos humanos, a la formación del pensamiento crítico y a la revalorización de los humanismos.

- Valoración: Este componente responde al cómo evaluar los contextos puestos en tensión con los campos teórico-prácticos, considerando la valoración, no como fin, sino como proceso formativo, lo que implica una exigencia en el desarrollo de reflexiones e instrumentos valorativos, que permitan la recuperación de experiencias formativas.

- Emergencias: Este campo está abierto a la construcción permanente, debe dar cuenta de las problemáticas, necesidades, expectativas, deseos y potencialidades que van surgiendo, no sólo en el aula de clase, sino también en el contexto y en la realidad de cada estudiante y sus entornos. Estas emergencias son importantes como recurso y eje generador de la práctica pedagógica generativa en espirales, que se nutren permanentemente.

- Proyecciones: Este componente responde a idealizaciones, estrategias o proyectos de aula que permitan que, el estudiante trascienda su actividad académica, y procure ser mediador de reflexión y trasformación en sus entornos desde los humanismos.

- Acción - reflexión: Este campo va dirigido a desarrollar la meta-cognición del proceso formativo por parte de toda la comunidad educadora, generando una praxis y una didaxis que dé cuenta de las reflexiones y las proyecciones en acciones concretas que aporten a las transformaciones requeridas.

- Recontextualización: Este componente implica valorar el ciclo, explícito en el desarrollo y construcción del currículo. En este sentido, se hace necesario volver sobre el diagnóstico situacional inicial y valorar los avances, los alcances, las emergencias, las prioridades y la proyección, para generar nuevas reflexiones colectivas del proceso y por ende estrategias de mejoramiento o fortalecimiento.

De ahí que las cartografias curriculares corresponden con un ejercicio de sistematización, mapeo y documentación de las prácticas formativas en relación con los cuatro componentes diseñados para la implementación del currículo crítico octagonal, donde se identifiquen: las emergencias, proyecciones, acciones-reflexiones y las re-contextualizaciones manifiestas en los procesos de formación del humanismo en Filosofia, Ética y Educación Religiosa.

A través de distintos instrumentos como la cartografia social... podemos construir conocimiento en el aula desde la propia experiencia. Lo producido por los participantes 
se relaciona con la teoría existente, generando un nuevo conocimiento teórico-práctico que permite a las personas intervenir en las modificaciones y cambiar las condiciones sociales y de conocimiento, de las cuales se partió (Valderrama, 2011, p. 3)

Esto es relevante en la medida en que se pretende construir desde la propia experiencia en el aula, de ahí que ésta cartografía curricular desde la propuesta del currículo crítico octagonal, implica dos momentos que responden a una doble naturaleza curricular (como diseño y como práctica): En su naturaleza de diseño, la propuesta curricular crítica octagonal se construye desde un colectivo académico, para poder definir los componentes de ese currículo, así mismo las configuraciones desarrollos y alcances de algunos de los componentes, que se corresponden con la naturaleza de diseño, aquellos componentes determinados fruto de las reflexiones, acuerdos y negociaciones del colectivo académico; Y la implementación del diseño curricular permite visibilizar y documentar las emergencias, proyecciones, acciones-reflexiones y las re-contextualizaciones manifiestas en la práctica pedagógica, para ser reflexionadas, con los estudiantes y a la par, en las sesiones de la Mesa de Humanismos entre el colectivo de docentes, segunda fase de esta investigación, de la cual se espera dar cuenta finalizando el año 2015.

\section{Para la discusión.}

El proyecto de investigación-acción en cuestión, ha permitido visualizar la necesidad y pertinencia en la construcción de un currículo crítico para el municipio de Rionegro, dado el contexto y la importancia de la formación en los humanismos desde un enfoque crítico. La Mesa de Humanismos (como estrategia de la investigación-acción) se ha consolidado como un escenario de reflexión y producción académica en el municipio, superando espacios de discusión, que se quedan en la palabra sin trascendencia, exigiendo no sólo una reflexión constante del quehacer de los docentes participantes, sino alcanzando en varios de ellos un nivel de praxis transformadora de su quehacer educativo.

Los maestros participantes del proyecto no son considerados objetos investigables, o sólo informantes, sino que se hacen actores dinámicos y activos, investigadores que socializan, debaten y resignifican sus propias prácticas y concepciones, a partir de la reflexión constante y el compartir de las mismas. Al hablar de currículo critico es importante la concienciación de que éste, trasciende la estandarización, el enfoque tradicional - técnico o historicista, posibilitando la construcción del mismo, no sólo de forma unidireccional, es decir, construcción que hace el maestro para su práctica, sino que es construcción reciproca, que involucra la comunidad, el contexto, los estudiantes y sus realidades.

Se identificaron claramente tres grandes enfoques curriculares que orientan desde el diseño, sus prácticas formativas en el humanismo: Currículos con enfoques historicistas, estandarizados y problematizadores, lo que indica por un lado una diversidad de opciones de la manera en que los maestros piensan sus diseños curriculares y a la par, las tendencias implícitas y explícitas que develan sus propios paradigmas de formación frente a las áreas del humanismo.

Las reflexiones que en el campo de la didáctica se alcanzaron, lograron ampliar los referentes de instrumentalización de la misma (desde las aproximaciones y el reconocimiento de los enfoques didácticos: general, diferencial, de las ciencias y crítico), que los maestros participantes compartían, desbordando en una abanico de posibilidades la didáctica crítica desde un ejercicio fundamental como ha sido, el Foro Intercolegial de Filosofia, el cual ha llevado más allá del aula de clase y de los campos de formación disciplinar, el humanismo 
y desde el cual se alcanzó un trabajo conjunto interinstitucional, del colectivo de maestros de la mesa y un material académico de reflexión donde los estudiantes de las diversas instituciones educativas del municipio, tienen la posibilidad de construir sus textos (con el acompañamiento de los maestros) y de expresarlos en el Foro con la participación de las comunidades educativas (estudiantes y maestros) del municipio de Rionegro, registradas en las memorias que se siguen consolidando como documentos de estudio y dinamización de lo social.

Las experiencias pedagógicas y didácticas significativas que fueron recuperadas desde los tres grupos focales (Filosofia, Ética y Educación Religiosa): Los foros (institucional e intercolegial) de filosofia, la construcción de disertaciones filosóficas, los encuentros de oratoria, los procesos de formación mediados por las Tecnologías de la Información y la Comunicación (TIC) y la experiencia de reajustes al plan de área de educación religiosa, son la posibilidad de visibilizar, compartir y reconstruir dichas experiencias, no solo desde las narrativas de los maestros, sino y además desde las prácticas de los maestros, como reconocimiento de los diversos enfoques didácticos: general, diferencial, de las ciencias y crítico.

Las implicaciones del diseño de este currículo crítico octagonal van desde las diferencias, negociaciones, acuerdos y desacuerdos alcanzados con los maestros participantes, que generó un movimiento de paradigmas en su fuero íntimo y sus concepciones, hasta lindar con sus prácticas formativas, la apertura en las posibilidades y la confrontación de sus nuevas perspectivas, frente a la construcción colectiva lograda en relación con los procesos educativos del humanismo.

Institucionalmente se presentaron implicaciones desde la participación de los maestros en el escenario de la Mesa de Humanismos, por los tiempos y la cotidianidad en los procesos que viven dichos centros educativos, hasta por las socializaciones y realimentaciones que los mismos maestros llevaban a las discusiones académicas y pedagógicas de los diferentes cuerpos colegiados, donde afloraron disertaciones en relación con la construcción y el diseño de los currículos, desde una metodología participativa, hasta la necesidad de implementar esta metodología en otras áreas de formación y claro está, como estrategia de las Mesas de Trabajo.

La propuesta sobre los componentes que configuran el currículo crítico octagonal, responde a la posibilidad de romper las lógicas verticales y horizontales que tradicionalmente se ha naturalizado en el diseño de los currículos. A la vez que plantear un diseño colectivo de los primeros cuatro componentes: contextualización, campos disciplinares, mediaciones y valoraciones, donde se puedan estructurar estos elementos fundamentales que orientaran los procesos de formación en los humanismos y deja abierta la construcción de los otros cuatro componentes: Emergencias, proyecciones, acciones-reflexiones y re-contextualizaciones, que posibilitan la documentación de las diversas dinámicas que se puedan presentar en la implementación del currículo crítico octagonal, para la consolidación de cartografias curriculares propias en cada caso.

Las cartografias curriculares son la posibilidad de visibilizar el mapa curricular que se genera, del diseño a su implementación, de lo planeado a lo emergente, de lo conocido a lo desconocido, de las conformaciones territoriales en cada campo de formación y su documentación a la reflexión continua con los estudiantes y claro está en la Mesa de Humanismos. Es recrear un ejercicio de sistematicidad de las prácticas formativas para la reflexión y consolidación del colectivo de maestros, como segunda fase de esta investigación en curso, durante todo el año 2015. 
La propuesta de un currículum crítico octagonal, no sólo es la posibilidad de cuestionar las formas tradicionales en las que se han venido naturalizando enfoques curriculares tecnicistas, con intencionalidades ocultas, desconocidas por los maestros, sino la oportunidad de abrir el horizonte comprensivo y proponer un ejercicio de innovación curricular, que emerge de ejercicios investigativos al interior de la mesa de humanismos y por ende gracias a la configuración de la comunidad académica pensante de la investigación-acción, como realidad y no sólo discurso, que transformo y amplio el marco de referencia de la manera como tradicionalmente se ha venido construyendo el diseño y las prácticas curriculares en el municipio.

En el presente año 2016, el proyecto se encuentra en su segunda fase de ejecución, en la cual y por medio de la estrategia de la Mesa de Humanismos con el colectivo de maestros, se está implementando el diseño del currículo crítico octagonal que fue diseñado en el año 2014-2015, que a la fecha está generando insumos en relación con los componentes de emergencias, proyecciones, acciones-reflexiones, re-contextualizaciones, para la continuidad en la reflexión y las validaciones de esta innovación educativa, en la formación de los humanismos en las diferentes instituciones educativas del municipio de Rionegro, con el propósito de presentar un segundo artículo en donde se expongan los resultados en la implementación del currículo crítico octagonal y las cartografias curriculares construidas, como ejercicio permanente de búsquedas en la consolidación del colectivo de maestros y de la pedagogía como campo de saber y poder. 


\section{Referencias bibliográficas.}

Klafki, W. (1991). Sobre la relación entre didáctica y metódica. Revista Educación y Pedagogía(5), 85-108. Obtenido de http://aprendeenlinea.udea.edu.co/revistas/index. $\mathrm{php} / \mathrm{revistaeyp/article/view/17024}$

Arias, J., Gómez, G., \& Osorio, E. (2008). Implicaciones de la pedagogía del texto en propuestas educativas. Rionegro: Divergráficas Ltda.

Camiloni, A., Davini, M., Edelstein, G., Litwin, E., Souto, M., \& Barco, S. (1997). Corrientes didácticas contemporáneas. Buenos Aires, Barcelona, México: Paidós.

Candelo, C., Ortiz, G., \& Unger, B. (2003). Hacer Talleres. Una guía práctica para capacitadores. Cali: Grafiq Editores.

Carmona, A. (2012). Memorias del I Foro institucional de Filosofía Librepensadores. Rionegro: Inédito.

Escobar, J., \& Bonilla, F. (2011). Grupos focales: Una Guía Conceptual y Metodológica. Cuadernos hispanoamericanos de psicologia, 9(1), 51-67.

Giroux, H. (1997). Los profesores como intelectuales: Hacia una pedagogía crítica del aprendizaje. España: Paidós.

Grundy, S. (1991). Producto o praxis del currículum. Madrid: Ediciones Morata.

Habermas, J. (2008). Teoría de la acción comunicatica. Crítica de la razón funcionalista (Vol. II). México: Taurus.

Hernández, G. (2014). Relatoría grupo focal con los docentes de educación religiosa. Rionegro: Inédito .

Hernández, G., Palacio, L., \& Pérez, F. (2014). 1 Evaluación de la mesa de humanismos. Rionegro: Inédito.

Hurtado, J. (2010). Metodología de la investigación. Guía para la comprensión holística de la ciencia. Venezuela: Ediciones Quirón.

Martínez, A., Noguera, C., \& Orlando, J. (2003). Currículo y modernización. Bogotá: Cooperativa Editorial Magisterio .

Muñoz Gaviria, D. (2011). El compromiso social de las facultades de educación: reflexiones pedagógicas en torno a la educación y la crisis de la modernidad. El Ágora USB, 11(1), 125-152. Obtenido de http://revistas.usb.edu.co/index.php/Agora/article/ view/390/149

Pérez, F. (2014). Construcción de un currículo crítico: Filosofia, ética y religión desde la mesa de humanismos. $X$ Encuentro de profesores de filosofia. Tunja: Universidad Pedagógica y Tecnológica de Colombia . 
Ramírez, A. (2013). Experiencia de reajustes al plan de área de educación religiosa en la institución educativa Gilberto Echeverri Mejía. Rionegro: Inédito.

Reale, G., \& Antiseri, D. (1998). Historia del pensamiento filosófico y científico. Barcelona: Editorial Heder.\}

Ríos, U. (2014). Memorias X versión del foro intercolegial de filosofía mito y religión. Rionegro: Litodosmil.

Sandoval Casilima, C. (2002). Investigación cualitativa. Bogotá: Instituto Colombiano para el Fomento de la Educacióm Superior ICFES - ARFO Editores . Obtenido de http:/ / aprendeenlinea.udea.edu.co/1ms / moodle/pluginfile.php/154268/mod_ resource/content/0/Metodologia_de_la_Investigacion/sandoval_Metodologia_de_la_ investigacion.pdf

Valderrama, H. (2011). El mapeo social como herramienta educativa en el trabajo por proyectos: Aprendizaje autónomo, activo e inductivo en la comunidad educativa. España: Universidad de Sevilla.

Zemelman, H. (2012). Pensar y poder: Razonar y grámatica del pensar histórico. México: Siglo XXI .

\section{Notas:}

1 Este artículo hace parte de la investigación "Construcción de un currículo crítico en filosofia, ética y educación religiosa" aprobado por la Dirección de Investigación y Desarrollo de la Universidad Católica de Oriente (UCO) en convocatoria IDFC.001.02 de 2013 